\title{
Sedation with Ketamine to Catch Up with a Failed Sedation using Midazolam
}

\author{
Raphaël Alluin*1, Johann Laangry ${ }^{1}$ and Elodie Andrée ${ }^{2}$ \\ ${ }^{1}$ Palliative Care Service, Nancy University Hospital, France \\ ${ }^{2}$ Department of Acute Geriatric Medicine, Metz-Thionville Regional Hospital, France \\ *Corresponding author: R Alluin, Nancy University Hospital, Palliative Care Service, France
}

ARTICLE INFO
Received: March 11, 2019
Published: ${ }^{\text {幽 March 18, } 2019}$
Citation: Raphaël A, Johann L, Elodie
A. Sedation with Ketamine to Catch
Up with a Failed Sedation using Mida-
zolam. Biomed J Sci \& Tech Res 16(1)-
2019. BJSTR. MS.ID.002791.

Keywords: Dyspnea; Deep Sedation; Midazolam; Ketamine; Palliative Care
ABSTRACT

Background: The problem of treatment-refractory symptoms is common in palliative care practice. In such circumstances, it is possible to sedate with midazolam. We propose here the case of a patient who benefited from deep sedation with ketamine as a rescue of a failed sedation with midazolam for refractory dyspnea.

Case presentation: Mrs. X, 76 years, with a weight of $50 \mathrm{Kg}$, was known since 2008 for breast cancer with lungs and liver metastasis. Only palliative care was delivered since one year. She was hospitalized in the geriatric department for pulmonary sepsis with bilateral pleural effusion. Because of a refractory dyspnea, it was decided, after discussion with the patient and his family, to start deep sedation using midazolam. Despite important midazolam doses, the comfort state of the patient was considered insufficient. With the support of the Mobile Palliative Care Team, deep sedation with ketamine was successfully carried out as a catch-up.

Discussion: It appears from experience that ketamine seems to be a good drug alternative in the event of the failure of deep sedation with midazolam, and especially a much simpler and more secure alternative to put in place in a routine care service than propofol sedation.

Conclusion: Ketamine appears to be a good alternative in case of midazolam sedation failure. This option is secure, manageable and easily accessible, unlike propofol. It is worth considering the conduct of clinical trials, given the interest of the palliative medical community in this therapeutic alternative in situations of midazolam sedation failure.

\section{Background}

The problem of treatment-refractory symptoms is common in palliative care practice. This phenomenon confronts the physician to his limits as a caregiver. When a satisfactory alternative is lacking, it's possible to propose proportionate sedation and if necessary deep sedation maintained until death. Currently the French Palliative Care Society (FPCS) recommendations' are to conduct a sedation following a specific protocol using midazolam [1]. The sedation intention has to be characterized with the SEDAPALL typology [2]. The midazolam is a manageable molecule but sometimes it is not possible to reach a deep enough level of sedation to relieve the patient. Some patients can present paradoxical effects or others will resist the sedative effect due to tachyphylaxis. As Sardin, said it in a paper published in 2017 [3] sometimes "midazolam fails". Some patients present resistance factors and require the use of a second line treatment. We think ketamine could an appropriate choice. At moderate to high doses ketamine presents sedative effect. Ketamine is a safe molecule preserving spontaneous breathing, swallowing reflex and keeping hemodynamic stability $[4,5]$. In fact itis safer to use in a routine care service than high doses of midazolam. We propose here the case 
of a patient who benefited from deep sedation with ketamine as a rescue of a failed sedation with midazolam for refractory dyspnea.

\section{Case Presentation}

Mrs. X, 76 years, with a weight of $50 \mathrm{Kg}$, was known since 2008 for breast cancer with lungs and liver metastasis. Only palliative care was delivered since one year. The patient lived at home with her husband. She was oxygen-dependent at $3 \mathrm{l} / \mathrm{min}$. Due to increasing dyspnea the patient was brought to the emergency by the Emergency medical service. After blood test and computed tomography scan the diagnosis of pulmonary sepsis with bilateral pleural effusion was retained. Because of the gravity of the situation, resuscitators were asked an advice. The patient was recused for intensive reanimation. Noninvasive treatment was decided with antibiotic and prioritization to comfort care. The patient was transferred in the geriatric department.

\section{Case Management}

Upon arrival at the geriatric department, oral sustained-release oxycodone was switched (120 mg morning and evening) for

intra-venous morphine $(160 \mathrm{mg} / \mathrm{d}$ with rescue doses of $5 \mathrm{mg}$ each hour if pain).No painful recrudescence happened. Quickly the patient respiratory status worsened leading to the necessity of an increase of treatment doses. Oxygen was increased from 3 to $5 \mathrm{l} / \mathrm{min}$. Despite introduction and increasing of midazolam to $1 \mathrm{mg} / \mathrm{h}$ the patient breathing remained uncomfortable. Facing this refractory dyspnea, and after discussion with the patient and her family, we decided to start deep sedation using midazolam, with a SEDAPALL typology D3P2C2 (sfap.org/system/files/sedapall_vf1.pdf). Midazolam was gradually increased to $10 \mathrm{mg} / \mathrm{h}$ with rescue doses of $7 \mathrm{mg}$ each hour "in case of necessity". Table 1 shows the detailed dosages of the mains treatments during the hospital stay. Despite important midazolam doses and the alteration of the patient's vigilance characterized by a RICHMOND Agitation-Sedation Scale score of -3 (any movement (but no eye contact) to voice), it persisted at external assessment symptoms of suffering due to the respiratory impairment. Facing this, the geriatric staff asked for an assessment from the Mobile Palliative Care Team (MPCT).

Table 1: Evolution of the dosages of the main symptomatic and sedative treatments in intra-venous.

\begin{tabular}{|c|c|c|c|c|c|c|c|c|}
\hline Treatments & & & j1 & j2 & j3 & j4 & j5 & j6 \\
\hline \multirow{4}{*}{ Midazolam } & Continuous dose & $\mathrm{mg} / \mathrm{kg} / \mathrm{jr}$ & 0 & 0,5 & 3,4 & 4,8 & 4,8 & 4,8 \\
\hline & \multirow{3}{*}{ Rescue dose } & Dose (mg) & 0 & 1 & 7 & 7 & 7 & 7 \\
\hline & & Number & 0 & 6 & 5 & 2 & 0 & 0 \\
\hline & & Refractory period (min) & / & 60 & 60 & 60 & 60 & 60 \\
\hline Kétamine & Continuous dose & $\mathrm{mg} / \mathrm{kg} / \mathrm{jr}$ & 0 & 0 & 0 & 2 & 3 & 3 \\
\hline \multirow{4}{*}{ Morphine } & Continuous dose & $\mathrm{mg} / \mathrm{kg} / \mathrm{jr}$ & 3.2 & 3.2 & 3.2 & 3.2 & 3.2 & 3.2 \\
\hline & \multirow[t]{3}{*}{ Rescue dose } & Dose (mg) & 5 & 5 & 5 & 5 & 5 & 5 \\
\hline & & Number & 2 & 2 & 2 & 1 & 0 & 0 \\
\hline & & Refractory period (min) & 60 & 60 & 60 & 60 & 60 & 60 \\
\hline \multirow[t]{2}{*}{ Paracetamol } & & dose $(\mathrm{mg} / \mathrm{j})$ & 4000 & 4000 & 4000 & 4000 & 4000 & 4000 \\
\hline & & dose splitting & 4 & 4 & 4 & 4 & 4 & 4 \\
\hline Scopolamine butylbromure & Continuous dose & $\mathrm{mg} / \mathrm{j}$ & 0 & 0 & 120 & 120 & 120 & 120 \\
\hline \multicolumn{9}{|c|}{ Symptoms scores } \\
\hline Sedation level & RICHMOND scale & & 3 & 2 & -3 & -4 & -5 & -5 \\
\hline \multirow{2}{*}{ Pain scale } & Algoplus scale & & / & I & $1-3 / 5$ & $0-1 / 5$ & $0-1 / 5$ & $0 / 5$ \\
\hline & Numerique scale & & $0-8 / 10$ & $0 / 10$ & / & / & / & / \\
\hline
\end{tabular}

\section{Case Outcome}

Upon considering the insufficient relief despite the midazolam sedation ongoing, was another molecule was proposed to deepen sedation. The FPCS recommendations [1] propose propofol as an alternative when midazolam failed. This molecule is particularly efficient but usually limited to anesthetic and reanimation uses. It requires the stewardship of a resuscitator or an anesthetist to obtain and use the treatment. This molecule also has depressive respiratory effects which is an important risk in routine care service with no cardiorespiratory monitoring or mechanical ventilation project. Considering all of this, ketamine was chosen and administered after discussion with the family to explain the situation, difficulties and the medical project. Aiming at deep sedation, ketamine was started at $2 \mathrm{mg} / \mathrm{Kg}$ per day intravenously using a syringe driver. At the same time non-symptomatic treatments and monitoring of vital constants were withdrawn. A decision was taken to adapt the 
treatment to the physical symptoms only. In parallel midazolam was kept at the same dose and scopolamine butylbromure was started at $120 \mathrm{mg} / \mathrm{d}$ intravenously. After 24 hours ketamine was increased to $3 \mathrm{mg} / \mathrm{Kg} /$ d, i.e. $150 \mathrm{mg}$ per day.

A re-assessment of the patient was performed 48 hours later by the MPCT. A proposal was made to increase ketamine to $4 \mathrm{mg} / \mathrm{Kg}$ per day meaning 200mg per day. After discussion between the MPCT, the family and the geriatric staff, we concluded that the patient's symptomatic equilibrium was satisfactory, basing our opinion on a RICHMOND Agitation-Sedation Scale score of -5 (no response to voice or physical stimuli). Ketamine was thus kept at $150 \mathrm{mg} / \mathrm{d}$. The patient's death was to occur the same evening, which is 48 hours after the introduction of ketamine.

\section{Discussion}

There are no clear guidelines for initiation of ketamine for deep sedation in palliative care. Ketamine is easy and safe to use (preserving spontaneous breathing and swallowing reflex) and its sedative effects are dose dependent. Used in second line or as a complement it seems to offer a satisfactory clinic efficacy for a deep sedation objective in refractory symptoms context. The limited scientific publications in this specific field as we said in a first paper [6] "is probably due to the difficulty of conducting experimental studies in a context such as terminal palliative care, and more particularly the question of sedation for refractory symptoms". However, some authors have already published similar case reports as Gil Z Shlamovitz [7], who like us used ketamine to conduct a deep sedation in a context of acute respiratory distress in the terminal phase ; or Carter's team [8] who performed a sedation with ketamine in rescue of a failed midazolam and barbiturates sedation for refractory symptom distress.

Given the possibilities offered by ketamine and the frequency of midazolam sedation failure alone, some authors propose a protocol for this use [3]. The doses proposed were higher than ours but in this case we kept midazolam ongoing. We think that the association of two or more drugs is perhaps a better way to conduct sedation in refractory symptoms situations, especially for resistant patients, than a unique all-purpose drug. It appears from experience that ketamine seems to be a good alternative in the event of a failure of deep sedation with midazolam and especially a much simpler and more secure alternative to put in place in a routine care service than propofol sedation. It also appears than lower doses can be used than what is proposed by other authors if ketamine is associated with another drug. Multimodal sedation is perhaps the key to success in these cases.

\section{Conclusion}

Ketamine appears to be a good alternative in case of midazolam sedation failure. This solution is secure, manageable and easily accessible unlike propofol. It is worth considering, given the interest that the palliative medical community has in this therapeutic alternative in situations of midazolam sedation failure, the conduct of clinical trials. These situations are difficult and the conduct of research seems tricky in this area but it is important to continue to improve our practices and the quality of care we provide.

\section{References}

1. (2017) Deep and continuous sedation maintained until death. Drug implementation. SFAP landmark.

2. (2017) Typology of palliative sedative practices at the end of life. SFAP.

3. Sardin B, Marsaud JP, Quesnel PA, Terrier G, Bourzeix JV, et al. (2018) When midazolam is no longer sufficient. Development and protocols of Limoges. Palliative Medicine 17: 148-166.

4. (2012) Ketamine and respiratory function. In: Mion G Ketamine, $2^{\text {nd }}$ (edn.), Arnette, p. 89-96.

5. (2012) Cardiovascular indications. In: Mion G. Ketamine, $2^{\text {nd }}(e d n$.$) ,$ Arnette, pp. 121-123.

6. Alluin R, Leheup BF, Piot E (2018) Refractory pain and sedationanalgesia with ketamine. Palliative Medicine 17: 232-236.

7. Shlamovitz GZ, Elsayem A, Todd KH (2013) Ketamine for palliative sedation in the emergency department. J Emerg Med 44(2): 355-357.

8. Carter M J, Gibbins J, Senior-Smith G, Thomas S, Guest P, et al. (2008) Ketamine: Does it have a role in palliative sedation? JPSM 36(4): 1-3.

\section{ISSN: 2574-1241}

DOI: 10.26717/BJSTR.2019.16.002791

R Alluin. Biomed J Sci \& Tech Res

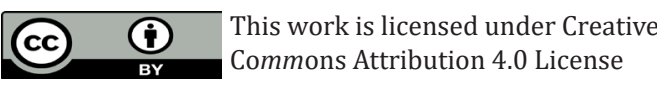

Submission Link: https://biomedres.us/submit-manuscript.php

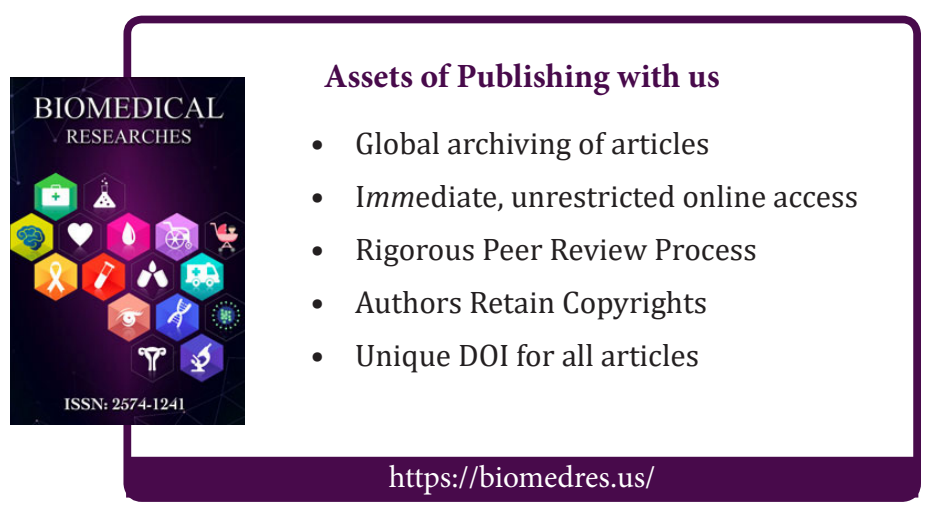

